\title{
Diagnosis and management of familial Mediterranean fever: Integrating medical genetics in a dedicated interdisciplinary clinic
}

\author{
Neda Zadeh, $M D^{1,2}$, Terri Getzug, $M D^{3}$, and Wayne W. Grody, MD, PhD ${ }^{1,2,4}$
}

\begin{abstract}
Familial Mediterranean fever is an autosomal recessive genetic disorder characterized by recurrent febrile polyserositis, especially prevalent in individuals of Mediterranean descent. Familial Mediterranean fever can have nonspecific manifestations that mimic many common acquired disorders such as infections, acute appendicitis, cholecystitis, and arthritis, which can delay diagnosis for many years and subject patients to extensive evaluations and even unnecessary surgery. Untreated familial Mediterranean fever can result in serious complications such as end-stage renal disease and malabsorption secondary to amyloid deposition in the kidneys and digestive tract, male and female infertility, and growth retardation in children. These significant sequelae, along with the episodic acute attacks, are readily preventable by treatment with oral colchicine and underscore the necessity of early detection and treatment from a medical, psychosocial, and economic standpoint. We describe our comprehensive approach to the accurate diagnosis and effective management of this disorder by means of a dedicated familial Mediterranean fever clinic that incorporates medical genetics on equal footing with general medicine. In addition to providing the clinician with the presenting features of familial Mediterranean fever, methods of diagnosis including molecular testing, and current management based on our extensive experience with hundreds of affected individuals, we also advance this approach as a model for the incorporation of medical genetics practice into the more traditional domains of general medicine. Genet Med 2011:13(3):263-269.
\end{abstract}

Key Words: familial Mediterranean fever, MEFV gene, peritonitis, periodic fever, colchicine

Camilial Mediterranean fever (FMF) is an autosomal reces- sive systemic autoinflammatory disorder characterized by recurrent episodes of fever accompanied by peritonitis, pleuritis, pericarditis, and/or arthritis, sometimes accompanied by an erysipelas-like skin rash. The condition was first described in 1945 under the name "benign paroxysmal peritonitis." $\mathrm{Al}$ though now known as FMF, this disorder may also be referred to as recurrent polyserositis, recurrent hereditary polyserositis, periodic disease, and periodic peritonitis. ${ }^{1}$ FMF has been established as a common condition among individuals of Mediterranean descent, with carrier rates as high as 1:7 in particular populations. ${ }^{1}$ In Arab countries, because of the high prevalence,

From the ${ }^{1}$ Department of Pathology and Laboratory Medicine, ${ }^{2}$ Department of Human Genetics, ${ }^{3}$ Division of Digestive Diseases, Department of Medicine, and ${ }^{4}$ Department of Pediatrics, UCLA Medical Center, Los Angeles, California.

Neda Zadeh, MD, UCLA Diagnostic Molecular Pathology Laboratory, 11633 San Vicente Blvd., Los Angeles, CA 90049. E-mail: nzadeh@gmail.com.

Disclosure: The authors declare no conflict of interest

Submitted for publication November 10, 2010.

Accepted for publication January 3, 2011.

DOI: $10.1097 /$ GIM.0b013e31820e27b1 it is considered a public health concern and has a significant effect on the health and welfare of children and adults. ${ }^{1}$

In contrast, the disease is considered rare in most Western countries, and many medical practitioners go through their entire career without ever encountering a case (or at least without diagnosing one!). Los Angeles is unusual in its extent of ethnic diversity, in particular its large populations of Armenian, Jewish, Arab, Iraqi, and Iranian immigrant groups. For that reason, our institution has long offered a unique clinic, originally established during the $1960 \mathrm{~s}$, that is dedicated to the diagnosis and treatment of patients with FMF. Over these years, the University of California at Los Angeles (UCLA) Familial Mediterranean Fever Clinic has accrued a patient experience of more than 700 individuals, providing us extensive clinical familiarity with this condition.

\section{HISTORICAL EPIDEMIOLOGY}

Although FMF has been reported in a variety of ethnic groups, the condition primarily affects populations of the Mediterranean basin: Arabs, Armenians, Turks, Greeks, Italians, Persians, and North African Jews (Morocco, Algeria, and Tunisia), as well as Sephardic and Ashkenazi Jews (from eastern Europe). ${ }^{2}$ Nonetheless, non-Mediterranean ancestry should not be assumed to exclude the diagnosis, as we and others have observed the condition in individuals of pure Japanese, Chinese, European, and South American descent. ${ }^{3,4}$ Conversely, there are areas such as sub-Saharan Africa, Ethiopia, Yemen, Scandinavian states, South Asia, India, and Thailand in which FMF has not been reported. A review conducted by Ben-Chetrit and Touitou $^{4}$ attempted to explain the origin of FMF in countries far from the Middle East and to ascertain whether the disorder in these non-Mediterranean countries differed in manifestation and prognosis.

Ben-Chetrit and Touitou analyzed the most frequent $M E F V$ mutations associated with particular ethnic groups. There are five common mutations (p.M694V, p.M694I, p.V26A, p.E148Q, and p.M680I) that are responsible for the majority of mutations in the Middle Eastern population of patients with FMF. Three of these mutations - E148Q, V726A, and M694V-are very ancient and first appeared 2500 years ago in Mesopotamia. During the 8th century, there was migration to Spain and North Africa (M694V and E148Q) by sailors who crossed the Mediterranean sea, or by land. Travel to Europe occurred as well (V726A), also by sea or land (Fig. 1). ${ }^{4}$ Countries with direct land connections, such as Armenia and Turkey, also experienced establishment of FMF into the population (Fig. 1). ${ }^{4}$

The "Chuetas" community in Palma de Mallorca are descendants of converted Jews who were expelled from Spain during the 11th century. This population possesses a considerable number of families with FMF. Affected Chuetas individuals seem to have phenotypic similarity to North African Jews with FMF who are also descended from Spain and were expelled from that country during the 16 th century. This observation is 


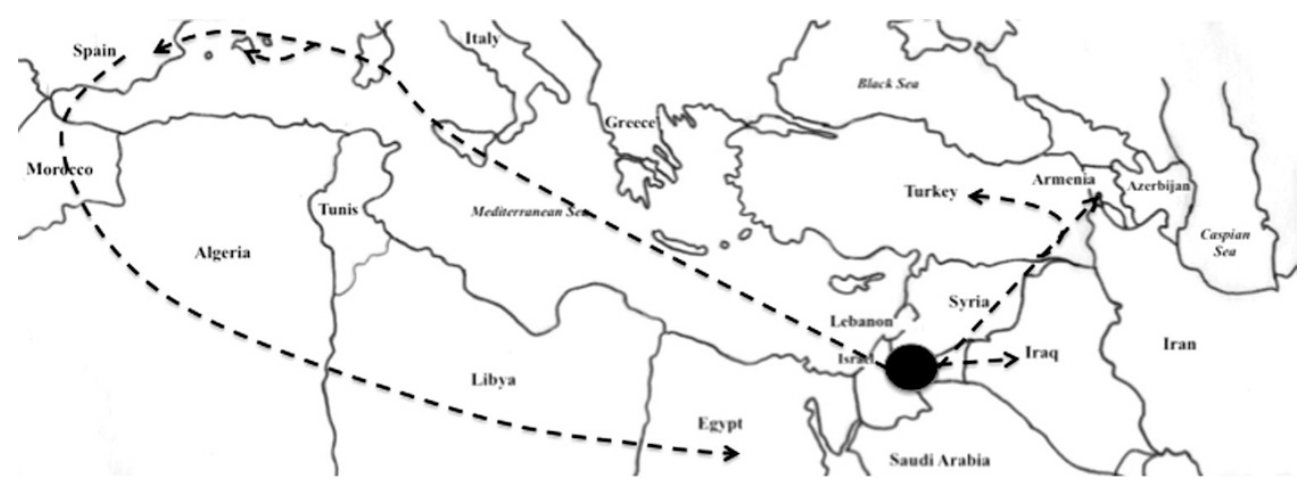

Fig. 1. Hypothesized route of migration of familial Mediterranean fever (FMF) founder mutations. Darkened circle marks the hypothesized origin of the three ancient FMF mutations. Dashed lines represent proposed migration of the founder mutations by land and sea migration. Adapted from Ben-Chetrit E, Levy M. Familial Mediterranean fever. Lancet 1998;351:659-664 and Ben-Chetrit E, Lerer I, Malamud E, Domingo C, Abeliovich D. The E148Q mutation in the MEFV gene: is it a disease-causing mutation or a sequence variant? Hum Mutat 2000;15:385-386.

suggestive of a common ancestor to both populations that most likely resided in Spain and traveled to the Middle East with the Muslims during the 8 th century. ${ }^{4}$

FMF has also been observed repeatedly in the Japanese population and poses a slightly greater challenge to trace the FMF mutation route to that country. Ben-Chetrit and Touitou ${ }^{4}$ suggested the possibility of the mutation traveling from Turkey to Japan by the Silk Road in a manner similar to Behcet disease. FMF mutations in Japan seem to consist mainly of M694I and $E 148 Q$, which also supports the theory of an introduced founder effect versus a de novo appearance. ${ }^{4}$

Just as FMF spread from the Middle East to Europe in ancient times, it has migrated to the New World in modern times (albeit by more modern modes of transport). North American communities of considerable size originating from Armenia (California), North Africa, and Middle Eastern countries, now reside in California and the East Coast. FMF families living in South America are mainly believed to be originally of Middle Eastern descent. ${ }^{4}$

\section{CLINICAL MANIFESTATIONS}

FMF classically presents with unprovoked, recurrent attacks of fever and painful polyserositis mainly affecting the peritoneum (most common), synovium, and pleura that usually (but not always) begin in childhood. ${ }^{1}$ The majority of affected individuals $(80 \%)$ present before 10 years of age and $90 \%$ before reaching the age of 20 years. Disease onset after the age of 40 years may represent a separate and milder disease entity, ${ }^{2}$ although responsive to the same treatment in our experience. Acute episodes may last from 24 to 72 hours and have variable frequency, often without a recognized triggering event. Some commonly reported precipitating causes include viral illness, emotional stress, excessive/intense physical activity, high-fat diet, extremes of temperature, and menstruation in women. The frequency of attacks may vary from once per week to once every 5-10 years, with the median frequency being approximately once a month. ${ }^{1}$ Approximately half of the affected individuals report experiencing a prodrome of discomfort and/or psychological uneasiness up to 24 hours before the actual attack.

Fever tends to coincide with arthritis and peritonitis symptoms; however, there have been documented FMF cases of fever without other symptomatology, especially in younger children (although admittedly, it is difficult for the young child to selfreport the nature of the discomfort). The febrile episodes are usually short-lived (2-4 days) with full spontaneous recovery but occasionally can last longer than 4 days and end unexpectedly. ${ }^{3}$ Our own clinical experience would suggest that this is very unusual; the fever is usually evanescent and does not persist as long as the other symptoms. Fevers are generally accompanied by abdominal (most common), chest, or joint pain; thus, the attacks resemble peritonitis, pleuritis, or synovitis (depending on the serous membrane involved) from other causes, which is the reason for frequent misdiagnosis, exploratory surgery, and unnecessary appendectomies. ${ }^{2}$

Joint pain and pleuritis are the second most common manifestations of FMF attacks, after acute abdominal pain. ${ }^{2}$ Articular pain may occur abruptly in an asymmetric or bilateral fashion involving large joints, most commonly the hip or knee followed by the ankle, shoulder, temporomandibular, or sternoclavicular joints. We observe in our patient population that the joint pain is usually monoarticular in each attack but may involve a different joint in subsequent attacks. It is not unusual for the arthritis to persist for several days or even longer after the other manifestations of the attack have subsided. ${ }^{5}$

Pleuritis may manifest as unilateral febrile dyspnea, with findings of diminished breath sounds and a pleural friction rub on the affected side. Pericarditis is rare but may present with retrosternal pain and ST abnormalities on electrocardiogram; it is often difficult to diagnose if concurrent with pleuritis. ${ }^{1}$ Both of these symptoms, although highly distressing, end as abruptly as they begin after the episode runs its course.

Skin manifestations include erysipelas-like lesions that are reminiscent of cellulitis and cluster unilaterally on the extensor surfaces of the leg or over the ankles and/or dorsum of the foot. ${ }^{1}$ The affected skin is generally erythematous and tender to palpation, with well-demarcated borders that can be $10-35 \mathrm{~cm}^{2}$ in area. ${ }^{5}$ The lesion can remain for 1-2 days and in our experience is much less common compared with the other symptoms of FMF.

The majority of patients with FMF (95\%) experience abdominal pain, which often seems remarkably similar to an acute abdomen by both history and physical examination. Peritoneal signs of board-like rigidity of the abdominal muscles, rebound tenderness, and abdominal distension with loss of bowel sounds are common findings during attacks. ${ }^{5}$ As mentioned earlier, in years past (less frequently at present owing to improved imaging technologies), it was not unusual for affected individuals to 
undergo unwarranted appendectomy based solely on clinical examination and laboratory findings (e.g., leukocytosis). This oversight could potentially be avoided if medical personnel obtain a thorough medical history (which will likely include description of similar episodes in the past), along with heightened suspicion based on patient ethnicity.

In untreated individuals, the risk of amyloidosis (SAA type) is arguably the most significant complication of this condition. Widespread amyloid deposition occurs in the kidneys primarily by the age of 40 years but can also affect the gastrointestinal tract, liver, spleen, thyroid, and in much later stages, the heart and testes as well. ${ }^{3}$ Renal insufficiency begins subtly but eventually progresses to frank nephrotic syndrome and ultimately end-stage renal disease requiring dialysis or renal transplantation. The mortality of FMF, a tragic outcome for this treatable disease, is almost always caused by renal failure. Certain ethnic backgrounds (North African Jewish, Turkish, and Armenian origin particularly from Yerevan, the capital of Armenia) are more predisposed to development of renal amyloidosis compared with other affected ethnic groups, ${ }^{3}$ whereas Ashkenazi Jews seem to be relatively protected. ${ }^{6}$ Serum amyloid levels can reportedly be elevated during attacks and symptom-free periods; however, this analyte is rarely assessed for either diagnostic purposes or clinical management. Other complications of untreated FMF can include decreased fertility by oligospermia/ azoospermia in men or by peritoneal inflammatory exudate leading to fibrous abdominal and ovarian adhesions noted in some affected women. ${ }^{5}$ These adhesions can also cause intermittent intestinal obstruction and complicate routine surgery in both men and women.

\section{DIAGNOSIS}

As FMF is an episodic disease found primarily in individuals of Mediterranean descent, the patient's medical history, family history, ethnicity, and physical examination findings are the most salient entities. During attacks, there is a massive influx of sterile polymononuclear leukocytes to affected sites in the body. Thus, serologic studies obtained during the episode typically reveal an increase in acute phase reactants such as C-reactive protein, fibrinogen, erythrocyte-sedimentation rate, and increased white blood cell count with neutrophilia. On a similar note, joint aspiration during attacks usually reveals a sterile pyogenic synovial effusion. Urinalysis tends to be normal; however, the finding of proteinuria should raise clinical concern for renal amyloidosis. ${ }^{1}$ In our experience, inflammatory serologies usually revert to normal between attacks, which may be a helpful diagnostic feature that distinguishes FMF from the more common autoimmune disorders. These clinical, historical, and laboratory findings are usually sufficient to make the presumptive diagnosis of FMF. Occasionally, the presentation is less typical, and in those cases, molecular genetic testing may be helpful.

\section{MOLECULAR GENETIC TESTING}

The marenostrin-encoding fever $(M E F V)$ gene is the only gene known to be associated with FMF and is located on chromosome $16 \mathrm{p} 13 . M E F V$ is $10 \mathrm{~kb}$ in size and comprises 10 exons, which generate a $3.7 \mathrm{~kb}$ transcript thought to be expressed in mature neutrophils and in fibroblasts derived from skin, peritoneum, and synovium, suggesting that it functions as an inflammatory regulator at the level of cytoskeletal organization. ${ }^{1,2}$ On an interesting note, several studies have suggested that individuals with FMF may have a decreased likelihood of developing atopic diseases compared with the general population (e.g., asthma and allergic rhinitis), ${ }^{7}$ which could theoretically be consistent with such a mechanism.

As $M E F V$ was independently identified in 1997 by two positional cloning consortia, the resulting 781 amino acid protein is known variously as marenostrin ("our sea" in reference to the Mediterranean focus of FMF) by the French FMF Consortium and as pyrin by the International FMF Consortium. ${ }^{8,9}$ Exons 10, 3, and 2 of $M E F V$ remain the major sites of disease-causing mutations (Fig. 2). Only minimal genotype/phenotype correlations have emerged, and we tend not to draw much attention to them with our patients, as we feel that the exact $M E F V$ mutation is not the sole determinant of clinical severity or colchicine response. ${ }^{2}$ Homozygosity for the $M 694 \mathrm{~V}$ mutation has been associated with relative resistance to colchicine, ${ }^{10}$ and we have observed this occasionally as well. Similarly, certain mutations are said to be more associated with amyloidosis than others. The E148Q mutation in exon 2, on the other hand, usually produces a mild or sometimes even silent phenotype, and there is ongoing debate whether it should be considered a true mutation versus a benign polymorphism. ${ }^{11}$ It is likely that there are unknown environmental factors or modifying genes that collaborate with alterations in $M E F V$ to produce a particular phenotype ${ }^{2}$; isoforms of the amyloid $S A A$ genes have been implicated as one possible factor. ${ }^{12}$ The normal function of pyrin is hypothesized to be involved in controlling inflammation by

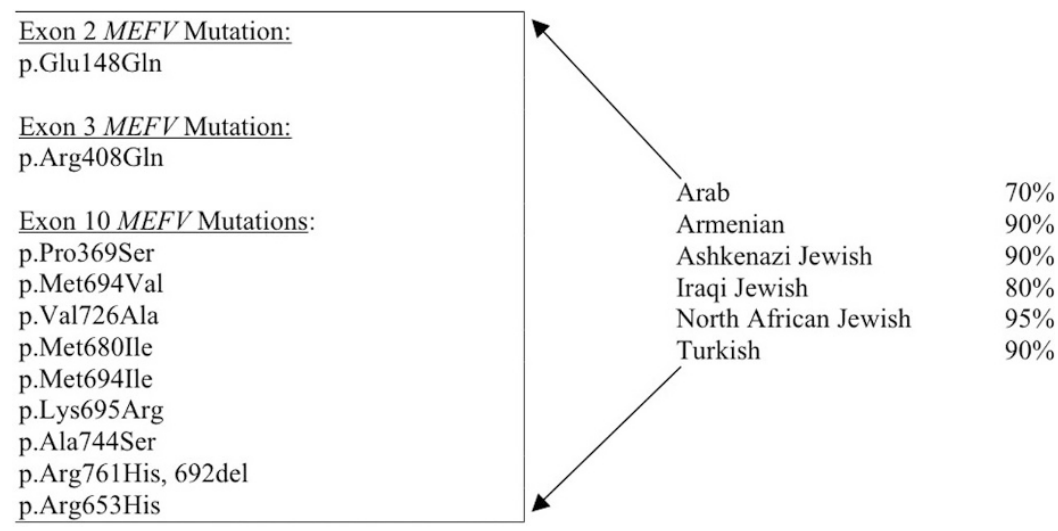

Fig. 2. Common MEFV mutations and their detection rate among particular ethnic groups. Modified from GeneTests. Familial Mediterranean fever. ${ }^{5}$ 
deactivating some aspect of the immune response. Lack of normal pyrin protein (as a result of disease-causing mutations in $M E F V$ ) would supposedly result in a full-scale inflammatory reaction of the serosal membranes, which is essentially an FMF attack ${ }^{13}$; hence, the classification of the disease as autoinflammatory as opposed to autoimmune.

Diagnostic molecular testing comprises different testing strategies depending on the ethnicity of the patient involved and the clinical situation. To date, more than 50 disease-causing mutations in $M E F V$ have been identified, with the majority being missense changes. ${ }^{14}$ The five most prevalent mutationsp.M680I, p.M694V, p.M694I, p.V726A (all in exon 10) and p.E148Q (exon 2) - are thought to be responsible for $>85 \%$ of patients with classic FMF. ${ }^{4,14}$ Our laboratory provides targeted molecular testing for the 12 most common $M E F V$ mutations (Fig. 2). This is typically offered as a first-tier approach, as the majority of affected individuals with classic FMF symptoms and Mediterranean ethnicity will most likely be homozygous or compound heterozygous for these common mutations (Fig. 3). If targeted mutation analysis does not reveal any disease-causing mutations or detects one pathogenic mutation rather than two, we then offer full sequence analysis of all the coding exons of the $M E F V$ gene if desired. It is generally left to the clinician's judgment whether the higher expense of molecular testing is the best option in pursuing the diagnosis as opposed to a therapeutic trial of colchicine (see later).

Current sequencing techniques will only detect point mutations and small insertions or deletions in coding regions of the gene. ${ }^{15}$ Thus, it is possible in individuals in which only one mutation is identified that a second disease-causing mutation may reside in a noncoding intronic area or in a gene regulatory region affecting messenger RNA splicing or expression. Fur-
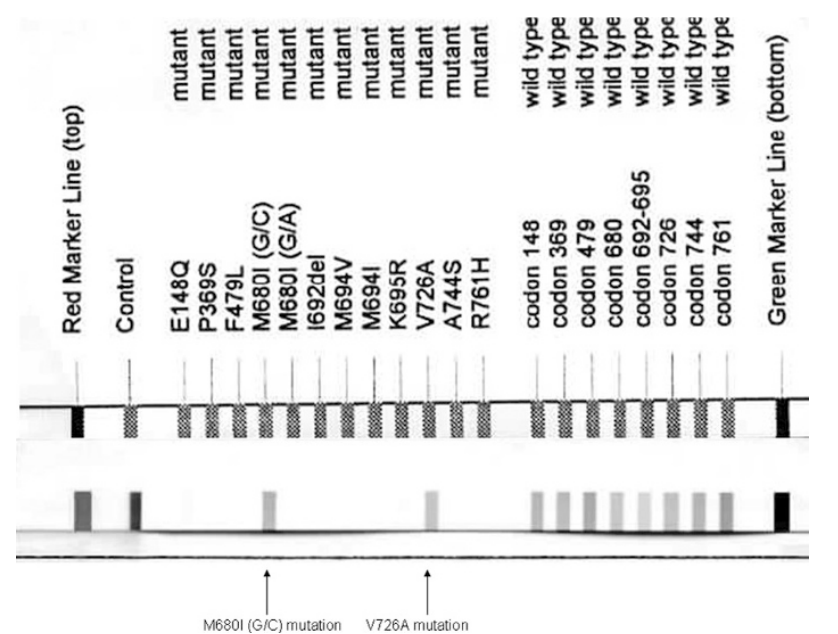

Fig. 3. Familial Mediterranean fever (FMF) strip assay: an example of targeted mutation analysis. This particular assay (ViennaLabs, Vienna, Austria) is designed to detect 12 common MEFV mutations by polymerase chain reaction and reverse hybridization. Mutations tested for in this particular assay include E148Q, P369S, F379L, M680I (G) C), M680I (G/A), I692del, M694V, M694I, K695R, V726A, $A 744 S$, and $R 761 \mathrm{H}$. Mutations screened in targeted mutation panels may vary among clinical laboratories. The patient sample shown has two heterozygous mutations (M860I [G/C] and V726A) confirming a (compound heterozygote) positive diagnosis of FMF in this patient. thermore, the medical literature suggests that single and multiexon $M E F V$ copy number changes (i.e., large deletions or duplications) do not contribute to the pathogenesis of FMF; for this reason, multiplex ligation-dependent probe amplification and other gene rearrangement techniques are not generally recommended for FMF testing. ${ }^{14,15}$

Molecular genetic diagnostic testing is used to provide a confirmation of the FMF diagnosis as it is obviously more specific than the other laboratory analytes such as erythrocyte-sedimentation rate and C-reactive protein. ${ }^{16}$ However, it is not uncommon, both in our experience and in the medical literature, to detect only a single-mutant allele in individuals of a high-risk ethnic background and presenting classic symptoms. Consequently, it is our observation that a significant proportion of patients have an incompletely explained clinical diagnosis from a molecular genetics standpoint. ${ }^{15}$ In such situations, we tend to accept the DNA findings as supportive of FMF and presume that the second mutation is not detected due to rarity or location outside the analyzed gene regions. ${ }^{2}$ This is analogous in many ways to situations that arise in both targeted and whole-gene analysis of CFTR mutations in patients who clinically seem to have cystic fibrosis. ${ }^{17}$

In the event that two mutations are not detected by either targeted mutation analysis or full gene sequencing in an individual with high clinical suspicion for FMF, another helpful diagnostic option is a 4-6-month trial of colchicine therapy to ascertain whether there is a decrease in frequency and severity of attacks. As the only two diseases that respond to colchicine are FMF and gout, a positive response essentially clinches the diagnosis, regardless of DNA findings. As the colchicine trial is safe and relatively inexpensive, it is often our preferred approach for diagnosis, rather than embarking on expensive DNA testing, which not infrequently proves inconclusive.

A question that often arises among our FMF families is whether to test asymptomatic family members, especially siblings of affected children. Molecular genetic testing of asymptomatic children is generally not encouraged on the grounds that FMF is a condition managed on symptomatology and ascribing to the ethical provision within the genetics community against DNA testing of healthy children. We make the case that the disease is readily treatable after symptoms appear, with no irreversible damage occurring before that time. In addition, we prefer to first observe the nature of the attacks in any affected child, so that we would know what we are treating. One argument raised against this approach is the possibility of so-called "silent" FMF, in which (hypothetically) an individual could be affected and vulnerable to organ damage from amyloidosis, in the absence of any overt pain and fever attacks. We are not convinced that such an entity (also designated as "Phenotype II") actually exists, although some centers have reported it. ${ }^{18,19}$ As a precaution, we do advise performing routine urinalysis at least yearly in any relatives we or the parents are concerned about, as the appearance of proteinuria would serve as an early screen for amyloid deposition.

\section{MANAGEMENT AND TREATMENT}

Treatment of FMF is centered on prevention of painful attacks and the development of amyloidosis. If attacks do occur, nonsteroidal antiinflammatory medication may be used to expectantly treat fever and pain, although it is not always effective. Before a definitive diagnosis, many patients with intractable pain are given steroids or narcotics; although these drugs do provide temporary relief, we do not recommend them for longterm use, for obvious reasons: aside from their well-known side effects, they are not useful in preventing the one fatal complication of FMF, amyloidosis. 
Colchicine is the gold standard and indeed the only recommended drug for treating FMF. It is thought to primarily concentrate in neutrophils and inhibit their increased chemotactic activity during FMF attacks. ${ }^{3} \mathrm{~A}$ study by Ozcakar et al. ${ }^{20}$ in 2010 concluded that apart from preventing acute episodes, colchicine also significantly improved height development and weight parameters in children with FMF.

Colchicine should be administered orally once the diagnosis of FMF is confirmed (or as a therapeutic trial in establishing the diagnosis). Adult dosing is $1.2-2.4 \mathrm{mg} / \mathrm{day}$, whereas children usually start at $0.3-1.2 \mathrm{mg} /$ day according to age and weight and can increase sequentially up to $2 \mathrm{mg}$ /day depending on how effectively the attacks are regulated. ${ }^{5}$ Paradoxically, very young children may sometimes require adult-level doses to show a response $\mathrm{e}^{21,22}$; the reason for this is not clear. In both children and adults, it may take some months of adjustment before the optimal dosage (evidenced by essentially complete prevention of attacks) is reached. The standard formulation in the United States is 0.6-mg tablets; in Europe and Israel, the strength is 0.5 $\mathrm{mg}$. It is acceptable for parents of young children to split the pills and/or crush them up into food. Doses can be taken at any time, with or without food. Some patients prefer once per day, whereas others experience better gastrointestinal tolerance by dividing the doses to twice or three times daily. It is crucial to drive home to patients (or their parents) that daily lifelong administration of colchicine is required to prevent both the fever/pain attacks and the silent amyloid deposition. We have observed that missing even a single dose often results in an acute attack within 24 to 72 hours, even in patients who have been under perfect control for years. ${ }^{3}$ Also, despite the common misconception, taking colchicine at the start of an attack is of no use; the drug will prevent attacks if taken continually but will not block an acute attack in progress.

If proteinuria is detected in a patient with FMF, more definitive renal studies are necessary, including a 24-hour urine protein assay and renal function tests (blood urea nitrogen and creatinine), and referral to a nephrologist for further work-up and management. Renal biopsy may be considered for adequate assessment of amyloid deposition and extent of parenchymal damage. A subcutaneous fat pad biopsy may also be used to diagnose systemic amyloidosis. ${ }^{23}$ Although cytogeneticists are familiar with colchicine's dramatic effect on cultured cells, patients (and parents) can be reassured that it is a very safe drug in vivo, at least in the doses used to treat FMF. Reported rare complications of colchicine use include myopathy, neuropathy, bone marrow suppression, and a toxic epidermal necrolysis-like skin reaction. It is not entirely clear to us how many of these cases were coincidental and not really related to the colchicine. Serious acute toxicities have invariably been the result of accidental overdose and inadvertent intravenous administration or untoward interactions with other drugs. ${ }^{24-26}$ Once patients are under good control on long-term colchicine therapy, we only require that they be evaluated by a physician annually and undergo a spot urinalysis for protein measurement and urine albumin to creatinine ratio (which we would expect to be negative). Although some practitioners recommend other monitoring tests such as liver enzymes and complete blood counts, we have not appreciated the need for them.

Considering colchicine's antimitotic effects, one might predict that it would be teratogenic, but in our opinion, there is no firm evidence to support that concern. ${ }^{27}$ As a precaution, we suggest that patients with FMF (both men and women) attempt to take a colchicine "holiday" starting approximately 3 months before attempting to conceive and continuing at least through the first trimester. However, in our experience, many of our patients find the resumption of attacks so intolerable (especially while trying to conceive) that they simply continue the drug. Moreover, in selected patients in whom infertility is an issue, and where the risk of miscarriage is high, colchicine may need to be continued on the premise that a febrile pain attack during pregnancy might jeopardize fetal outcome. To date, we have not observed any adverse pregnancy outcomes or birth defects in our large patient cohort (T. Getzug, unpublished data). In fact, it is well known that attacks are usually suppressed naturally during pregnancy in more than $50 \%$ of affected women. It may be prudent in cases when colchicine was used during conception or early pregnancy to recommend fetal ultrasound to provide reassurance. Whether to also offer amniocentesis, with its attendant risks, remains an open question. A recent study by Ben-Chetrit et al. ${ }^{28}$ in Israel found no difference in fetal outcome or incidence of birth defects between affected women who either continued taking or abstained from colchicine during pregnancy. They also pointed out that the common practice in Turkey (where FMF is quite prevalent) is not to recommend amniocentesis for prenatal colchicine exposure. ${ }^{28}$

\section{INTEGRATED MEDICAL GENETICS/GENERAL MEDICINE APPROACH TO FMF DIAGNOSIS AND MANAGEMENT: THE UCLA MODEL}

From the foregoing description, it should be evident that FMF, although nominally a single-gene Mendelian disorder, in many ways behaves as a complex disease, replete with variable expressivity, and affecting many organ systems by poorly understood autoinflammatory mechanisms. Indeed, FMF, similar to the other periodic fever syndromes familial Hibernian fever and hyper IgD syndrome, reflects a complex derangement of the "inflammasome"- the sum total of interacting genes and proteins involved in the inflammatory response. Moreover, as its major symptoms - acute abdominal pain, fever, arthritis, etc.are nonspecific and could potentially signal countless other medical and surgical conditions, patients with FMF are usually initially encountered by general pediatricians, internists, emergency medicine physicians, etc.; in fact, many never see a medical geneticist, whether or not properly diagnosed. That is not the case, however, at our institution.

UCLA has a large clinic dedicated to the diagnosis and treatment of patients with FMF. Since the early 1960s, the clinic has served as a major referral center for this underrecognized but very treatable condition. It is the largest clinic of its kind in the Western hemisphere, with more than 700 registered patients. Some of the original clinical research studies investigating the pathogenesis and treatment of FMF, before colchicine became the standard of care, were conducted at UCLA by Drs. Arthur Schwabe and Sherman Mellinkoff, the cofounders of the clinic. Because these physicians specialized in gastroenterology, the clinic's home has always been within the Division of Digestive Diseases in the Department of Medicine, and author T.G., also a gastroenterologist, is the current medical director of the clinic. Certainly, this is a benefit in view of the common abdominal presentation of the disease, which can be confused with irritable bowel syndrome, inflammatory bowel disease, celiac disease, and other disorders familiar to gastroenterologists. However, approximately 10 years ago, it was presciently recognized that genetic issues were also central to the care of these patients, and senior author W.W.G., who was already offering testing for $M E F V$ mutations in UCLA's molecular diagnostic laboratory, was invited to join the group as a regular attending physician. $\mathrm{He}$, in turn, incorporated the clinic into the rotation schedule for 
all our medical genetics fellows and molecular genetics fellows (including first author N.Z.), just as it already had been for gastroenterology fellows, rheumatology fellows, internal medicine residents, and medical students. The interdisciplinary nature of the clinic is further enhanced by the presence of Dr. Claire Panosian, representing the subspecialty of Infectious Disease (which is often invoked in the differential diagnosis of FMF), along with ready access to rheumatologists who share the same clinic space. The only constraint in this arrangement is that being based in adult medicine, the clinic's nursing staff are not comfortable dealing with pediatric patients, so provisions have been made that all children younger than 16 years who are referred for FMF evaluation are seen in our Medical Genetics Clinic (by W.W.G.). This is especially beneficial if other pediatric specialty services are needed, as the pediatric immunologists and rheumatologists happen to share the same clinic space. Moreover, there is continual crossreferral and consultation between the adult and pediatric clinics.

It should be evident from the foregoing discussion just how many difficult issues come up in the diagnosis and management of FMF that can be addressed uniquely by geneticists. The variable expressivity of the disorder; the remarkably high carrier frequency in some ethnic groups (e.g., Armenians) causing the appearance of (pseudo-)dominant inheritance;29 the often inconclusive or even misleading nature of $M E F V$ gene analysis; and the decisions about when to pursue genetic testing and/or treatment, especially in young children-all these issues arise from the genetics of the disorder. In addition, more pointed questions often come up from patients and their families: Why is this disease so common in my ethnic group? How can I have the disease when you found only a single mutation? Does the mutation(s) you detected predict how severe my condition will be? How can I be the only person affected in my family? What is our recurrence risk if we have more children? Will our younger child eventually get the disease? What will happen when my affected child has children of her own?

Regarding recurrence risks, we always take time to explain the autosomal recessive mode of inheritance of the condition, just as would transpire in any genetic counseling session. We presume that both parents of an affected child are obligate carriers. Carrier testing is carefully discussed with the family; we explain that the only real objective of such testing would be to identify the parental mutations for use in subsequent prenatal testing in a future pregnancy. Although we do mention carrier testing as a potential option to all our younger couples, we have never observed a single instance, among our many hundred of FMF families, when a couple requested prenatal diagnosis with the intent to terminate an affected fetus. Our patients and their families tend to view FMF as a completely treatable and manageable condition and, therefore, generally do not think about trying to prevent it in this way.

Finally, FMF represents an ideal model disease for this type of integrated subspecialty approach, harnessing the expertise of medical genetics, gastroenterology, rheumatology, and other multidisciplinary involvement for appropriate medical management and activism, especially as it is an eminently treatable condition. An oft-heard lament among the medical genetics community is that we too often serve primarily as diagnosticians but then have little to offer therapeutically for most of the disorders we diagnose. FMF, however, is, although not curable, completely alleviative to the point that diagnosed patients can live essentially normal lives, with normal life expectancies, careers, and child bearing (whereas undiagnosed patients continue to suffer and die needlessly). Furthermore, the treatment of FMF does not entail expensive, difficult, or esoteric enzyme replacement, recombinant gene therapy, specialized diet, or synthesized small molecular ligand inhibitor treatment, but rather a common, generic drug that has been around for centuries. Still, it demands accurate diagnosis and some experience with the disorder to prescribe effectively. In this sense, the medical geneticist serves as both diagnostic and therapeutic expert, whether in a consulting role or as primary provider. We might add parenthetically that the inexpensive and generic nature of colchicine has recently come into conflict from a branded and Food and Drug Administrationapproved formulation called Colcrys ${ }^{\circledR}$ that is up to 50 times more expensive; there is concern that this will present an unexpected financial burden on patients with FMF who must take the drug every day for life. ${ }^{30}$

For all these reasons, FMF has proven, at least in our experience in Los Angeles, to be a highly appropriate disorder for delivery of dedicated clinical services in close partnership between medical genetics and the primary internal medicine or pediatric specialists. However, it is by no means the only such disorder amenable to such a model. We can envision numerous other complex neurogenetic, cardiac, neoplastic, gastroenterologic, and other disorders that would benefit from such a coordinated and comprehensive approach; and the number will only increase in the coming years as whole-genome analysis yields many new genetic markers conferring risk for such disorders. These developments offer tremendous opportunity for the expansion and refocusing of medical genetics practice, well beyond our traditionally circumscribed areas of dysmorphology, developmental problems, and inborn errors of metabolism.

\section{REFERENCES}

1. El Shanti H, Majeed HA, El-Khateeb M. Familial Mediterranean fever in Arabs. Lancet 2006;367:1016-1024.

2. Telatar M, Grody WW. Molecular genetic testing for familial Mediterranean fever. Mol Genet Metab 2000;71:256-260.

3. Ben-Chetrit E, Levy M. Familial Mediterranean fever. Lancet 1998;351: 659-664.

4. Ben-Chetrit E, Touitou I. Familial Mediterranean fever in the world. Arthritis Rheum 2009;16:1447-1453.

5. Shohat M, Halpern GJ. GeneTests. Familial Mediterranean fever. Available at: www.genetests.org. Accessed November 1, 2010.

6. Lidar M, Kedem R, Berkun Y, Langevitz P, Livneh A. Familial Mediterranean fever in Ashkenazi Jews: the mild end of the clinical spectrum. $J$ Rheumatol 2010;37:422-425.

7. Sackesen C, Bakkaloglu A, Sekerel BE, et al. Decreased prevalence of atopy in pediatric patients with familial Mediterranean fever. Ann Rheum Dis 2004;63:187-190.

8. French FMF Consortium. A candidate gene for familial Mediterranean fever. Nat Genet 1997; 17:25-31.

9. International FMF Consortium. Ancient missense mutations in a new member of the RoRet gene family are likely to cause familial Mediterranean fever. Cell 1997;90:797-807.

10. Soylemezoglu O, Arga M, Fidan K, et al. Unresponsiveness to colchicine therapy in patients with familial Mediterranean fever homozygous for the M694V mutation. J Rheumatol 2010;37:182-189.

11. Ben-Chetrit E, Lerer I, Malamud E, Domingo C, Abeliovich D. The E148Q mutation in the MEFV gene: is it a disease-causing mutation or a sequence variant? Hum Mutat 2000;15:385-386.

12. Jéru I, Hayrapetyan H, Duquesnoy P, et al. Involvement of the modifier gene of a human Mendelian disorder in a negative selection process. PLoS One 2009;4:e7676.

13. Samuels J, Aksentijevich I, Torosyan Y, et al. Familial Mediterranean fever at the millennium. Clinical spectrum, ancient mutations, and a survey of 100 American referrals to the National Institutes of Health. Medicine (Baltimore) 1998; 77:268-297.

14. Booty MG, Chae JJ, Masters SL, et al. Familial Mediterranean Fever with a Single $M E F V$ mutation. Where is the second hit? Arthritis Rheum 2009;60: 1851-1861.

15. van Gijn ME, Soler S, de la Chapelle C, et al. Search for copy number alterations in the $M E F V$ gene using multiplex ligation probe amplification, experience from three diagnostic center. Eur J Hum Genet 2008;16:1404-1406.

16. Brik R, Shinawi M, Kepten I, Berant M, Gershoni-Baruch R. Familial Mediterranean fever: clinical and genetic characterization in a mixed population of Jewish and Arab patients. Pediatrics 1999;103:e70. 
17. Moskowitz SM, Chmiel JF, Sternen DL, et al. Clinical practice and genetic counseling for cystic fibrosis and CFTR-related disorders. Genet Med 2008; 10:851-868.

18. Balci B, Tinaztepe K, Yilmaz E, et al. MEFV gene mutations in familial Mediterranean fever phenotype II patients with renal amyloidosis in childhood: a retrospective clinicopathological and molecular study. Nephrol Dial Transplant 2002; 17:1921-1923.

19. Kutlay S, Yilmaz E, Koytak ES, et al. A case of familial Mediterranean fever with amyloidosis at the first manifestation. Am J Kidney Dis 2001:38:E34.

20. Ozcakar ZB, Kadioglu G, Siklar Z, et al. The effect of colchicine on physical growth in children with familial Mediterranean fever. Eur J Pediatr 2010; 169:825-828.

21. Ozkaya N, Yalçinkaya F. Colchicine treatment in children with familial Mediterranean fever. Clin Rheumatol 2003;22:314-317.

22. Kallinich T, Haffner D, Niehues T, et al. Colchicine use in children and adolescents with familial Mediterranean fever: literature review and consensus statement. Pediatrics 2007;119:e474-e483.

23. van Gameren I, Hazenberg BPC, Bijzet J, et al. Amyloid load in fat tissue reflects disease severity and predicts survival in amyloidosis. Arthritis Care Res 2010;62:296-301.
24. Maxwell MJ, Muthu P, Pritty PE. Accidental colchicine overdose. A case report and literature review. Emerg Med $J$ 2002;19:265-367.

25. Bonnel RA, Villalba ML, Karwoski CB, Beitz J. Deaths associated with inappropriate intravenous colchicine administration. J Emerg Med 2002;22: 385-387.

26. Rollot F, Pajot O, Chauvelot-Moachon L, Nazal EM, Kélaïdi C, Blanche P. Acute colchicine intoxication during clarithromycin administration. Ann Pharmacother 2004;38:2074-3077.

27. Ben-Chetrit E, Ben-Chetrit A, Berkun Y, Ben-Chetrit E. Pregnancy outcomes in women with familial Mediterranean fever receiving colchicine: is amniocentesis justified? Arthritis Care Res 2010;62:143-148.

28. Ben-Chetrit E, Ben-Chetrit A, Berkun Y, Ben-Chetrit E. Pregnancy outcomes in women with familial Mediterranean fever receiving colchicine: is amniocentesis justified? Arthritis Care Res 2010;62:143-148.

29. Caglar MK, Altugan FS, Ozyurt H, Atasoy HI. Screening of family members of children with familial Mediterranean fever: true-autosomal and pseudoautosomal inheritance. Acta Rheumatol Port 2008;33:415-420.

30. Grody WW, Getzug T. Colchicine's other indication: effect of FDA action (Letter). N Engl J Med 2010;363:2267-2268. 